\title{
Free Vibration of Axially Inhomogeneous Beams that are Made of Functionally Graded Materials
}

\author{
Yong Huang and Hai-Wu Rong \\ Department of Mathematics, Foshan University, Foshan, Guangdong 528000, China
}

\begin{abstract}
(Received 16 November 2014; accepted: 8 October 2015)
A simple approach to deal with an engineering problem of free vibration of functionally graded beams with nonuniform cross-sections where the material properties arbitrarily vary along the axial direction is presented. Instead of directly solving the fourth-order governing differential equation with variable coefficients, we transform the governing equation to a system of linear algebraic equations based on the polynomial expansion and integral technique. Then, a characteristic equation in natural frequencies will be obtained. Several examples of estimating natural frequencies for axially graded beams and non-uniform beams are presented, which show that our method has a fast convergence and the obtained numerical results are accurate. The proposed method is important to investigate because of the dynamic behavior of axially non-uniform beams in engineering applications.
\end{abstract}

\section{INTRODUCTION}

Non-homogeneous structural components with axially varying material properties are common in buildings, bridges, machine parts. There has been a great amount of research dedicated to investigating the dynamic behaviors of axially nonuniform beams. In this field, many approximate methods or numerical techniques have been formulated, which include Rayleigh's quotient method, the finite element method, the finite difference method, the boundary element method, and the differential quadrature method. By assuming that the beam's cross sectional area and the moment of inertia is arbitrary to polynomial functions of the position, Abrate ${ }^{1}$ used the Rayleigh-Ritz approach and Lagrange multiplier method to analyze the free vibration of nonuniform beams with the general shape and arbitrary boundary conditions. Analytical solutions of the vibration for the nonuniform beam with cross section geometries with exponentially variable widths have been obtained for three different types of boundary conditions associated with simply supported, clamped and free ends. $^{2}$ Nachum and Altus used the functional perturbation method to determine the natural frequencies and mode shapes of non-homogeneous rods and beams. ${ }^{3}$ By applying the Adomian modified decomposition method, Hsu et al. converted the governing differential equation to a recursive algebraic equation and kept the boundary conditions within simple algebraic frequency equations which were suitable for symbolic computation. ${ }^{4}$ Based on the fact that a nonuniform beam can be partitioned into multi homogeneous uniform sub-beams, Singh et al. ${ }^{5}$ developed a numerical method for determining the natural frequencies of a nonuniform beam. By assuming the material constituents are varied throughout the thickness or longitudinal directions according to a simple power law, Alshorbagy et al. used the numerical finite element method to investigate the dynamic behaviors of functionally graded beams. ${ }^{6}$ Based on the homotopy perturbation method and variational iteration method, Coskun and co-workers investigate the free vibration and buckling behaviors of beam. ${ }^{7-10}$

Clearly, most of the aformentioned methods do not yield a closed-form solution, but rather numerical or approximate results. It is well-known that when the graded elasticity and/or cross section of the beam varies along the axial direction, a problem of solving a differential equation with nonuniform coefficients based on the Euler-Bernoulli assumption arises. Therefore, closed-form solutions for free vibration of nonuniform beams are often difficult to find. So far, few analytical solutions are found in arbitrary gradient change due to the difficulty of mathematical treatment. Fortunately, by making use of the semi-inverse method, Elishakoff and co-workers treated a large class of problems involving graded beams of special forms such as polynomials and obtained explicit fundamental frequency of free vibration. ${ }^{11-14}$ Aydogdu ${ }^{15}$ also used the semi-inverse method to study the vibration and buckling of simply supported beams. However, the semi-inverse method cannot apply for graded beams of any axial inhomogeneity. By using the differential transform method, Catal investigated the problem of free vibration of one end fixed and the other simply supported beam resting on elastic soil. ${ }^{16}$ The free vibration and stability of axially functionally graded tapered Euler-Bernoulli beams have been studied by Shahba and Rajasekaran ${ }^{17}$ through solving the governing differential equations based on the differential transform element method. Based on the EulerBernoulli theory and Haar wavelet approach, Hein and Feklistova $^{18}$ investigated the free vibration nonuniform and functionally graded beams with various boundary conditions and varying cross sections. The free vibration and stability analysis of axially functionally graded tapered Timoshenko beams were carried out using the finite element approach. ${ }^{19}$ Recently, $\mathrm{Li}$ et. $\mathrm{al}^{20}$ investigated the free vibration of exponential functionally graded beams, where the characteristic equations in closed form with different boundary conditions have been derived.

The objective of this paper is to present a simple approach for analyzing the dynamic behavior of axially functionally graded and nonuniform beams, where the material properties are varying arbitrarily along the axial direction. By expanding the mode shapes as power series, we transform the governing differential equation with variable coefficients to a system of algebraic equations with unknown coefficients. The natural frequencies can be easily determined from the existing condition of a nontrivial solution in the resulting system. We will apply the method to evaluate the natural frequencies of uniform homogeneous and nonuniform beams with the kinds of boundary conditions. Moreover, the effects of the gradient parameter and cross section on the natural frequencies under various end supports will be discussed. 


\section{FORMULATION AND METHOD}

A functionally graded and/or nonuniform beam of length $L$, which is subject to the action of transverse loading, is considered. Based on the Euler-Bernoulli beam theory, the governing differential equation for free vibration of axially graded and nonuniform beams can be written as: ${ }^{11}$

$$
\frac{\partial^{2}}{\partial x^{2}}\left[D(x) \frac{\partial^{2} w}{\partial x^{2}}\right]+m(x) \frac{\partial^{2} w}{\partial t^{2}}=q(x, t)
$$

where $x, w$, and $q$ are the axial coordinate, deflection, and distributed transverse loading, respectively; $D(x)=E(x) I(x)$ is the flexural rigidity of the beam, which is a function of the axial coordinate $x$, and depends upon both Young's modulus $E(x)$ and the second moment of cross sectional area $I(x)$; and $m(x)=\rho(x) A(x)$ is the mass of the beam per unit length, which depends upon the cross sectional area $A(x)$ and mass density $\rho(x)$. We introduce a non-dimensional coordinate $\xi=x / L$ and consider harmonic vibration so that $w$ is represented as:

$$
w(x, t)=W(x) e^{i \omega t} .
$$

By placing Eq. (2) into Eq. (1) and then assuming $q(x, t)=0$, one can get:

$$
\frac{d^{2}}{d \xi^{2}}\left[D(\xi) \frac{d^{2} W}{d \xi^{2}}\right]-k m(\xi) W=0, \quad 0 \leq \xi \leq 1
$$

with $D(\xi)=E(\xi L) I(\xi L), k=\omega^{2} L^{4}$. By avoiding to solve the differential Eq. (3) directly, we introduce a new and simpler method $^{21}$ to investigate the free vibration of axially inhomogeneous beams. Now, we take an axially graded and nonuniform clamped-pinned (C-P) beam with clamped end at $\xi=0$ and a simply supported end at $\xi=1$ for example. Therefore, the corresponding boundary conditions read:

$$
\begin{aligned}
& W=0, \quad \frac{d W}{d \xi}=0, \quad \xi=0 ; \\
& W=0, \quad \frac{d^{2} W}{d \xi^{2}}=0, \quad \xi=1 .
\end{aligned}
$$

Actually, the problem becomes solving a set of a fourthorder ordinary differential governing Eq. (3) combining with the boundary conditions given by Eqs. (4) and (5). First of all, we expand $W(\xi)$ as a power series. Or rather, if it neglected a sufficiently small error, the unknown $W(\xi)$ can be approximately expanded as:

$$
W(\xi)=\sum_{i=0}^{N} \frac{c_{i} \xi^{i}}{i !}, \quad 0 \leq \xi \leq 1 ;
$$

where $c_{i}$ are unknown coefficients and $N$ is a certain positive integer, which is chosen and should be large enough so that the rest have a negligible error. By using the clamped conditions at $\xi=0$, then substituting Eq. (6) into Eq. (4), one gets:

$$
\begin{aligned}
& c_{0}=0 ; \\
& c_{1}=0 .
\end{aligned}
$$

Bearing Eq. (5) in mind, the application of the pinned condition $\xi=1$ to Eq. (6) leads to:

$$
\begin{aligned}
c_{0}+c_{1}+\frac{c_{2}}{2 !}+\ldots+\frac{c_{N}}{N !} & =0 \\
c_{2}+c_{3}+\frac{c_{4}}{2 !}+\ldots+\frac{c_{N}}{(N-2) !} & =0 .
\end{aligned}
$$

It is easily found that in order to solve the linear Eqs. (7) to (10), other $N-3$ independent equations are needed for uniquely determining $c_{i}$. This can be achieved by using the following method. ${ }^{21}$ Inserting Eq. (6) into the resulting differential Eq. (3) for each case leads to:

$$
\begin{gathered}
\sum_{i=0}^{N}\left[C_{i}^{4} D(\xi)+2 C_{i}^{3} \xi \frac{d D(\xi)}{d \xi}+C_{i}^{2} \xi^{2} \frac{d^{2} D(\xi)}{d \xi^{2}}\right] \frac{\xi^{i-4}}{i !} c_{i}- \\
k \sum_{i=0}^{N} \frac{m(\xi) \xi^{i}}{i !} c_{i}=0
\end{gathered}
$$

where $C_{i}^{4}=i(i-1)(i-2)(i-3), C_{i}^{3}=i(i-1)(i-2)$, $C_{i}^{2}=i(i-1)$. We multiply both sides of Eq. (11) by $\xi^{t}$ $(t=0,1,2, \ldots N-4)$ and then integrate it with respect to $\xi$ between 0 and 1 , which yields another $N-3$ linear algebraic equations of unknown coefficients $c_{i}$ :

$$
\sum_{i=0}^{N}\left(f_{j i}-k g_{j i}\right) c_{i}=0, \quad j=4,5,6, \ldots N
$$

with

$$
f_{j i}=\int_{0}^{1}\left[C_{i}^{4} D(\xi)+2 C_{i}^{3} \xi \frac{d D(\xi)}{d \xi}+C_{i}^{2} \xi^{2} \frac{d^{2} D(\xi)}{d \xi^{2}}\right] \frac{\xi^{j+i-8}}{i !} d \xi
$$$$
g_{j i}=\int_{0}^{1} \frac{\xi^{j+i-4}}{i !} m(\xi) d \xi \text {. }
$$

Consequently, making use of Eqs. (7) to (10) and (12), we can obtain a system of linear algebraic equations for unknown coefficient $c_{i}$, which can be rewritten in a compact form as $\left(\mathrm{F}_{n n}-k \mathrm{G}_{n n}\right) \mathbf{C}_{n}=0, \mathrm{~F}_{n n}=\left(f_{i j}\right)_{(N+1) \times(N+1)}$, $\mathbf{G}_{n n}=\left(g_{i j}\right)_{(N+1) \times(N+1)}, \mathbf{C}_{n}=\left(c_{i}\right)_{(N+1) \times 1}$. To obtain a nontrivial solution of the resulting system, the determinant of the coefficient matrix of the system has to vanish, so we immediately get a characteristic equation in eigenvalues $k$ :

$$
\operatorname{det}\left(\mathrm{F}_{n n}-k \mathrm{G}_{n n}\right)=0 .
$$

By inspecting the above procedure, if $D(\xi)$ and $m(\xi)$ are given functions, one can readily find that the obtained corresponding characteristic Eq. (14) has multi-roots, since it is a polynomial in natural frequency $k$. Therefore, a numerical procedure to look for its roots is simple with the aid of commercial software. As a result, both the lower and higher natural frequencies of axially nonuniform beams can be determined simultaneously from the multi-roots.

By using this method, we can easily deal with the problem for all kinds of boundary conditions. In the case of cantilever or clamped-free (C-F) beams, substituted Eq. (6) into the corresponding boundary conditions can aid in obtaining the first four linear equations for $c_{i}$. It is only needed to replace Eqs. (7) to (10) for the newly obtained linear equations while keeping the last $N-3$ equations as the same form of Eq. (12). There is no doubt that the introduced method can give rise to great simplification of dealing with the free vibration of axially functionally graded nonuniform beams for different boundary conditions. It should be noted that besides the expansion of Eq. (6) of $W(\xi)$, other expansion approximations of the unknown $W$ such as Chebyshev polynomial, trigonometric functions, etc., are also applicable. It is well known that the first 
Table 1. The first three non-dimensional natural frequencies $\Omega_{n}$ for uniform beams.

\begin{tabular}{|c|c|c|c|c|c|c|c|}
\hline & & & \multicolumn{7}{c|}{ Case A } & Chebyshev polynomial & Case B & \multirow{2}{*}{ Exact $^{22}$} \\
& $n$ & $N=6$ & $N=10$ & $N=14$ & $N=14$ & $N=14$ & \\
\hline \multirow{3}{*}{ C-F } & 1 & 3.517076 & 3.516015 & 3.516015 & 3.516015 & 3.516015 & 3.5160 \\
& 2 & 22.233362 & 22.034492 & 22.034492 & 22.034492 & 22.034492 & 22.0345 \\
& 3 & 118.144436 & 61.715119 & 61.697215 & 61.697215 & 61.697260 & 61.6972 \\
\hline & 1 & 5.604273 & 5.593321 & 5.593321 & 5.593321 & 5.593321 & 5.5933 \\
C-G & 2 & 30.996978 & 30.225861 & 30.225848 & 30.225848 & 30.225848 & 30.2258 \\
& 3 & 132.954050 & 74.692768 & 74.638884 & 74.638884 & 74.639024 & 74.6389 \\
\hline & 1 & 9.872527 & 9.869604 & 9.869604 & 9.869604 & 9.869604 & 9.8696 \\
P-P & 2 & 50.199602 & 39.479138 & 39.478418 & 39.478418 & 39.478453 & 39.4784 \\
& 3 & 131.812493 & 88.848180 & 88.826440 & 88.826440 & 88.822660 & 88.8264 \\
\hline & 1 & 15.547849 & 15.418206 & 15.418206 & 15.418206 & 15.418206 & 15.4182 \\
C-P & 2 & 66.389739 & 49.968013 & 49.964862 & 49.964862 & 49.964871 & 49.9649 \\
& 3 & 179.002422 & 104.382769 & 104.247702 & 104.247702 & 104.247325 & 104.2477 \\
\hline \multirow{3}{*}{ C-C } & 1 & 22.564206 & 22.373286 & 22.373285 & 22.373285 & 22.373285 & 22.3733 \\
& 2 & 91.651514 & 61.688324 & 61.672823 & 61.672823 & 61.672866 & 61.6728 \\
& 3 & 223.362612 & 121.106105 & 120.903400 & 120.903400 & 120.899064 & 120.9034 \\
\hline
\end{tabular}

Table 2. The first three non-dimensional natural frequencies $k_{n}$ for axially graded P-P beams.

\begin{tabular}{|c|c|c|c|c|c|c|c|c|c|}
\hline \multirow{2}{*}{$E_{\text {ratio }}$} & \multirow[b]{2}{*}{$n$} & \multicolumn{2}{|l|}{$\alpha=0$} & \multicolumn{2}{|c|}{$\alpha=0.2$} & \multicolumn{2}{|c|}{$\alpha=1$} & \multicolumn{2}{|c|}{$\alpha=5$} \\
\hline & & Alshorbagy et al. ${ }^{6}$ & Present & Alshorbagy et al. ${ }^{6}$ & Present & Alshorbagy et al. ${ }^{6}$ & Present & Alshorbagy et al. ${ }^{6}$ & Present \\
\hline \multirow{3}{*}{0.25} & 1 & $2.2214(2.2203)$ & 2.221441 & $2.4118(2.4106)$ & 2.411854 & $2.7546(2.7533)$ & 2.754715 & $3.0849(3.0834)$ & 3.085017 \\
\hline & 2 & $4.4425(4.4338)$ & 4.442883 & $4.847(4.8374)$ & 4.847367 & $5.484(5.473)$ & 5.484458 & $6.0756(6.0636)$ & 6.076306 \\
\hline & 3 & $6.6631(6.6338)$ & 6.664324 & $7.2792(7.247)$ & 7.280589 & $8.2123(8.1753)$ & 8.213893 & $9.0664(9.0262)$ & 9.068501 \\
\hline \multirow{3}{*}{0.5} & 1 & 2.6417 & 2.641754 & 2.7271 & 2.727195 & 2.9119 & 2.911949 & 3.1067 & 3.106839 \\
\hline & 2 & 5.2831 & 5.283508 & 5.4681 & 5.468500 & 5.8162 & 5.816727 & 6.158 & 6.158589 \\
\hline & 3 & 7.9238 & 7.925262 & 8.2076 & 8.209143 & 8.7199 & 8.721549 & 9.2114 & 9.213255 \\
\hline \multirow{3}{*}{1} & 1 & 3.1415 & 3.141593 & 3.1415 & 3.141593 & 3.1415 & 3.141593 & 3.1415 & 3.141593 \\
\hline & 2 & 6.2827 & 6.283185 & 6.2827 & 6.283185 & 6.2827 & 6.283185 & 6.2827 & 6.283185 \\
\hline & 3 & 9.423 & 9.424778 & 9.423 & 9.424778 & 9.423 & 9.424778 & 9.423 & 9.424778 \\
\hline \multirow{3}{*}{2} & 1 & 3.7359 & 3.736004 & 3.6671 & 3.667188 & 3.4628 & 3.462911 & 3.1939 & 3.193902 \\
\hline & 2 & 7.4714 & 7.472008 & 7.3183 & 7.318925 & 6.9167 & 6.917292 & 6.4611 & 6.461511 \\
\hline & 3 & 11.206 & 11.208013 & 10.968 & 10.970467 & 10.37 & 10.371728 & 9.7163 & 9.717998 \\
\hline \multirow{3}{*}{4} & 1 & 4.4428 & 4.442882 & 4.3166 & 4.316696 & 3.8957 & 3.895756 & 3.2684 & 3.268399 \\
\hline & 2 & 8.885 & 8.885765 & 8.6022 & 8.603071 & 7.7555 & 7.756194 & 6.7036 & 6.704037 \\
\hline & 3 & 13.326 & 13.328649 & 12.886 & 12.888748 & 11.614 & 11.616200 & 10.105 & 10.106382 \\
\hline
\end{tabular}

Table 3. The first three non-dimensional natural frequencies $\Omega_{n}$ for P-P and C-C graded beams.

\begin{tabular}{||c|c|c|c|c|c|c|c|}
\hline & & \multicolumn{2}{|c|}{ Case A } & \multicolumn{2}{c|}{ Case B } & \multicolumn{2}{c|}{ Case C } \\
& $n$ & Present & Exact $^{13}$ & Present & Exact $^{13}$ & Present $^{13}$ & \multicolumn{2}{c|}{ Exact $^{13}$} \\
\hline & 1 & 18.973666 & $18.973666\left(\Omega_{1}^{2}=360\right)$ & 28.982753 & $28.982753\left(\Omega_{1}^{2}=840\right)$ & 67.970582 & $67.970582\left(\Omega_{1}^{2}=4620\right)$ \\
P-P & 2 & 74.540233 & - & 114.560860 & - & 273.106343 & - \\
& 3 & 167.167790 & - & 257.124973 & - & 612.707077 & - \\
\hline & 1 & 41.270740 & - & 63.894272 & - & 147.358557 & - \\
C-C & 2 & 114.760318 & - & 177.140992 & - & 816.762224 & - \\
& 3 & 225.830912 & - & 348.131592 & - & 824.427049 & - \\
\hline
\end{tabular}

kind of Chebyshev polynomials can be obtained by the following recurrence relations:

$$
\begin{aligned}
T_{0}(z) & =1, \quad T_{1}(z)=z, \\
T_{i+1}(z) & =2 z T_{i}(z)-T_{i-1}(z) ;
\end{aligned}
$$

where $z$ is over the interval $[-1,1]$. By introducing variable substitution $\xi=(z+1) / 2$, we can easily get the Chebyshev polynomials over the interval $[0,1]$ :

$$
\begin{aligned}
T_{0}^{*}(\xi) & =1, \quad T_{1}^{*}(\xi)=2 \xi-1, \\
T_{i+1}^{*}(\xi) & =2(2 \xi-1) T_{i}^{*}(\xi)-T_{i-1}^{*}(\xi) .
\end{aligned}
$$

As a result, the unknown $W(\xi)$ can be expanded into the shifted Chebyshev polynomials in general over the interval $[0,1]$ as:

$$
W(\xi)=\sum_{i=0}^{N} c_{i} T_{i}^{*}(\xi), \quad 0 \leq \xi \leq 1 .
$$

By substituting Eq. (17) to the governing equation and corresponding boundary conditions, we can easily determine the natural frequency based on the Chebyshev polynomial. And the procedures are omitted here.

\section{NUMERICAL RESULTS AND DISCUSSION}

In order to examine the effectiveness of the proposed method, let us first consider the case of a homogeneous beam with uniform cross sections. In this case, $D(\xi)=E I$ and $m(\xi)=\rho A$ are unchanged, and the non-dimensional natural frequencies $\Omega=\omega L^{2} \sqrt{\rho A / E I}$ can be exactly calculated from the following frequency equations: ${ }^{22} \mathrm{P}-\mathrm{P}, \sin \sqrt{\Omega}=0$; C-C, $\cos \sqrt{\Omega} \cosh \sqrt{\Omega}-1=0$; C-F, $\cos \sqrt{\Omega} \cosh \sqrt{\Omega}+1=0$; C-P, $\tan \sqrt{\Omega}-\tanh \sqrt{\Omega}=0$; and C-G, $\tan \sqrt{\Omega}+\tanh \sqrt{\Omega}=0$. It should be noted that the above frequency equations are highly nonlinear except for the P-P beams and that it is not easy to find an analytical solution for any assumed stiffness parameter. However, based on the aforementioned approach, the numerical results of natural frequencies for any modes can be easily calculated. For checking the convergence of the suggested method, we have calculated non-dimensional natural frequencies $\Omega$ of a homogeneous beam with different boundary conditions by taking different $N$ values in Eq. (6). Evaluated results of first three non-dimensional natural frequencies $\Omega_{n}$, and the exact ones are tabulated in Table 1. By comparing our numerical results with the exact ones, it is clear from Table 1 that the 
numerical results have a rapid convergence. With $N$ increasing from 6 to 14, the errors between the numerical and exact results dramatically decrease, which indicates that the present approach is very efficient. As a result, a higher accuracy can be achieved through increasing $N$. Furthermore, we have calculated the non-dimensional natural frequencies by using Chebyshev expansions in Eq. (17) with $N=14$. Seen from Table 1, the results based on two different expansions are identical to each other up to 6 decimal digits. Through observing the linear equations in Eq. (12), one may find that those equations can be obtained in another manner, such as multiplying both sides of Eq. (11) by $\xi^{2 j}$ and changing $j$ from 2 to $N-2$, while keeping the first four linear Eqs. (7) to (10) the same. For clarity, we denote the former way as Case A and the latter as Case B. From the numerical results, we can discover that treatment has little influence on the natural frequencies although the last $N-4$ linear equations are changed, which indicates that the behaviors of free vibration are mostly determined by the end supports in nature. Therefore, we will choose the procedure of Case A with $N=14$ in the following computation.

Next, we consider a functionally graded beam with axial inhomogeneity, where the cross sectional area $A$ and moment of inertia $I$ are unchanged. This is of benefit to design of nonhomogeneous beams for certain purposes. To show the effect of the inhomogeneity on the natural frequencies, the material characteristics (such as Young's modulus, mass density, etc.) can be assumed to be varied a power law distribution as: ${ }^{6}$

$$
P(\xi)=P_{R}+\left(P_{L}-P_{R}\right)(1-\xi)^{\alpha} ;
$$

where $P_{L}$ and $P_{R}$ are the corresponding material properties of the left $(\xi=0)$ and the right side $(\xi=1)$ of the beam, respectively, and $\alpha$ is the non-negative power-law exponent parameter describing the volume fraction change of both constituents involved through the axis of the beam. Based on the introduced method, the effects of Young modulus ratio $E_{\text {ratio }}=E_{L} / E_{R}$ and the gradient parameter $\alpha$ on first three dimensionless natural frequencies $k_{n}=\sqrt{\omega L^{2} \sqrt{\frac{\rho_{R} A}{E_{R} I}}}$ are displayed in Table 2 with $N=14$ for simply supported boundary conditions. The free vibration characteristics and dynamic behavior of a functionally graded beam for axially material distributions as a power law form Eq. (18) have been analyzed numerically by Alshorbagy et al. through using the finite element method. ${ }^{6}$ We should note that the results in parentheses cited from the work of Alshorbagy et al. ${ }^{6}$ were calculated from slenderness ratio $L / h=20$, while others were obtained with $L / h=100$. By comparing the proposed model with published work, ${ }^{6}$ we can find that the present numerical results agree reasonably well with existing results with $L / h=100$. However, the accuracy between the present and existing results drops with the ratio $L / h=20 .^{6}$ This phenomenon indicates that the EulerBernoulli theory is suited for slender beams. In addition, the fundamental frequencies are also sensitive to the Young modulus ratio and the gradient parameter $\alpha$, as expected. From Table 2, the natural frequencies increase with an increase in the power exponent (when $E_{\text {ratio }}<1$ ) and decrease with an increase in the power exponent (when $E_{\text {ratio }}>1$ ). For a constant power exponent, an increase in $E_{\text {ratio }}$ causes the increase in fundamental frequencies. For comparison, here we also list numerical results of the well-known first three natural frequencies $k_{n}$ of homogeneous beams $\left(E_{\text {ratio }}=1\right)$ with simply end conditions: $3.1415926,6.2831853,9.4247780$. Our results are identical to the exact ones up to 7 decimal digits for homogeneous beams.

For axially graded beams with a uniform cross section, Elishakoff and Guede presented closed-form solutions to vibrating non-homogeneous beams by the semi-inverse method. ${ }^{13}$ For comparison, we take the mass density $\rho$ and Young's modulus $E$ as polynomial functions for three cases: Case A, $\rho(\xi)=\rho_{0}, E(\xi)=E_{0}\left(3+3 \xi-2 \xi^{2}-2 \xi^{3}+\xi^{4}\right)$; Case B, $\rho(\xi)=\rho_{0}, E(\xi)=E_{0}\left(\frac{31}{3}-6 \xi^{2}+\xi^{4}\right)$; and Case C, $\rho(\xi)=$ $\rho_{0}\left(\frac{26}{165}+\xi^{2}\right), E(\xi)=E_{0}\left(\frac{7337}{270}-\frac{52}{3} \xi^{2}+\frac{13}{54} \xi^{4}+\xi^{6}\right)$. Elishakoff and Guede ${ }^{13}$ first preselected polynomial functions serve as an exact mode shape of the inhomogeneous beam then solved the semi-inverse problem of finding the distributions of the stiffness and the inertial term. For simply supported beams, they determined the results of exact first-order nondimensional natural frequency $\Omega_{1}^{2}=\omega_{1}^{2} L^{4} \rho_{0} A / E_{0} I$, which are identical to $360,840,4620$ for Case A, B and C, respectively. Based on the present approach, the numerical results of $\Omega_{n}=\omega_{n} L^{2} \sqrt{\rho_{0} A / E_{0} I}$ for P-P and C-C graded beams and those of the first three modes are listed in Table 3. From Table 3, we find that the results of first-order of non-dimensional natural frequency for P-P beams are the same as the exact one. ${ }^{13}$ Other natural frequencies for the arbitrary order vibration mode for $\mathrm{C}-\mathrm{C}$ beams are also obtained, but they seem not to be derived in closed form by the inverse method. ${ }^{13}$

In what follows, we discuss the free vibration of EulerBernoulli beams with a nonuniform cross section along the length direction, where the material properties $E$ and $\rho$ of the beams keep constants. Firstly, we take the cross sectional area $A$ and moment of inertia $I$ as $A=A_{0}(1+\beta \xi)^{4}$, $I=I_{0}(1+\beta \xi)^{4}$, where $\beta$ is a geometrical parameter, and $A_{0}$ and $I_{0}$ are the cross sectional area and the second moment of cross sectional area of the beam at $\xi=0$, respectively. For this case, Abrate ${ }^{1}$ derived the exact solution of Eq. (3) as

$$
\begin{gathered}
W=\frac{1}{(1+\beta \xi)^{2}}\left[\gamma_{1} \sin \left(\sqrt{\Omega_{n}} \xi\right)+\gamma_{2} \cos \left(\sqrt{\Omega_{n}} \xi\right)+\right. \\
\left.\gamma_{3} e^{\sqrt{\Omega_{n}} \xi}+\gamma_{4} e^{-\sqrt{\Omega_{n}} \xi}\right]
\end{gathered}
$$

where $\Omega_{n}=\omega_{n} L^{2} \sqrt{\rho A_{0} / E I_{0}}$ are the non-dimensional natural frequencies, and the constants $\gamma_{i}(i=1, \ldots, 4)$ are to be determined from the boundary conditions. Therefore, we can easily obtain the characteristic equations for kinds of boundary conditions applying the support ends to Eq. (19). Based on the introduced method, the first four non-dimensional natural frequencies $\Omega_{n}=\omega_{n} L^{2} \sqrt{\rho A_{0} / E I_{0}}$ for tapered beams of C-P, $\mathrm{P}-\mathrm{P}$, and $\mathrm{C}-\mathrm{C}$ ends are evaluated in Table 4 with different values for parameter $\beta$. The exact results derived previously by Abrate $^{1}$ are also presented in Table 4 . It is should be noted that the exact results for C-P end are cited from Abrate's publication, ${ }^{1}$ and other results for P-P and C-C boundary conditions are calculated based on the exact corresponding characteristic equations. From Table 4, it is seen that our numerical results show excellent consistency with the above-mentioned results. In particular, our numerical results of the first three natural frequencies are nearly identical to the results acquired by Abrate. However, even for the fourth-order natural frequency, the errors between two results are less than $0.004 \%$. Moreover, we can find that the geometrical parameter $\beta$ plays an important role in determining the natural frequencies for C-P and P-P beams. However, the natural frequencies of clamped-clamped 
Table 4. Non-dimensional natural frequencies $\Omega_{n}$ for tapered beams $A / A_{0}=I / I_{0}=(1+\beta \xi)^{4}$.

\begin{tabular}{||c|c|c|c|c|c|c|c||}
\hline \multirow{2}{*}{ Support ends } & $n$ & \multicolumn{2}{|c|}{$\beta=0$} & \multicolumn{2}{|c|}{$\beta=1$} & \multicolumn{2}{c|}{$\beta=2$} \\
& & Abrate $^{1}$ & Present & Abrate $^{1}$ & Present $^{1}$ & Abrate $^{1}$ & Present $^{*}$ \\
\hline \multirow{4}{*}{ C-P } & 1 & 15.4182 & 15.418206 & 12.3635 & 12.363516 & 10.5984 & 10.598379 \\
& 2 & 49.9649 & 49.964862 & 47.6265 & 47.626496 & 46.6678 & 46.667829 \\
& 3 & 104.248 & 104.247702 & 102.025 & 102.024580 & 101.174 & 101.173899 \\
& 4 & 178.270 & 178.277198 & 176.105 & 176.107010 & 175.304 & 175.304062 \\
\hline \multirow{5}{*}{ P-P } & 1 & 9.8696 & 9.869604 & 9.1757 & 9.175715 & 8.2900 & 8.290058 \\
& 2 & 39.4784 & 39.478418 & 40.1156 & 40.115623 & 40.8774 & 40.877363 \\
& 3 & 88.8264 & 88.826439 & 89.8680 & 89.868021 & 91.2205 & 91.220566 \\
& 4 & 157.9137 & 157.916169 & 159.1757 & 159.175845 & 160.9028 & 160.902625 \\
\hline C-C & 1 & 22.3733 & 22.373285 & 22.3733 & 22.373285 & 22.3733 & 22.373291 \\
& 2 & 61.6728 & 61.672823 & 61.6728 & 61.672823 & 61.6728 & 61.672857 \\
& 3 & 120.9034 & 120.903400 & 120.9034 & 120.903479 & 120.9034 & 120.903638 \\
\hline
\end{tabular}

Table 5. The first mode of non-dimensional natural frequencies $\Omega_{1}$ for $\mathrm{C}-\mathrm{C}$ beams.

\begin{tabular}{|c|c|c|c|c|c|c|}
\hline$c_{h}$ & $c_{b}$ & 0 & 0.2 & 0.4 & 0.6 & 0.8 \\
\hline \multirow[t]{2}{*}{0} & Shahba et al. ${ }^{23}$ & 20.4721 & 20.4152 & 20.2883 & 20.0186 & 19.3845 \\
\hline & Present & 20.472088 & 20.415143 & 20.288273 & 20.0185778 & 19.384355 \\
\hline \multirow[t]{2}{*}{0.2} & Shahba et al. ${ }^{23}$ & 18.2170 & 18.1996 & 18.1286 & 17.9437 & 17.4566 \\
\hline & Present & 18.216992 & 18.199537 & 18.128557 & 17.943601 & 17.456370 \\
\hline \multirow[t]{2}{*}{0.4} & Shahba et al. ${ }^{23}$ & 15.8282 & 15.8498 & 15.8350 & 15.7367 & 15.4025 \\
\hline & Present & 15.828146 & 15.849733 & 15.834917 & 15.736555 & 15.402140 \\
\hline \multirow[t]{2}{*}{0.6} & Shahba et al. ${ }^{23}$ & 13.2293 & 13.2896 & 13.3319 & 13.3238 & 13.1529 \\
\hline & Present & 13.229150 & 13.289418 & 13.331634 & 13.323402 & 13.152117 \\
\hline \multirow[t]{2}{*}{0.8} & Shahba et al. ${ }^{23}$ & 10.2235 & 10.3229 & 10.4255 & 10.5168 & 10.5339 \\
\hline & Present & 10.221839 & 10.321203 & 10.423581 & 10.514532 & 10.530924 \\
\hline
\end{tabular}

beams are the same regardless of the parameter $\beta$. This is due to the fact that the tapered beams for clamped-clamped beams with properties varying as in this example will have the same governing differential equation and the same boundary conditions as the uniform beam. ${ }^{1}$

Finally, free vibration of axially graded tapered beams with a nonuniform cross section is concerned. Not only the effective material properties of mass density and Young's modulus, but also the cross section area and moment of inertia vary continuously along the axial direction. Here, the distribution of mass density $\rho(\xi)$ and Young's modulus $E(\xi)$ are assumed to be polynomial functions:

$\rho(\xi)=\rho_{0}\left(1+\xi+\xi^{2}\right), \quad E(\xi)=E_{0}(1+\xi), \quad \xi \in[0,1] ;$

where $\rho_{0}$ and $E_{0}$ are the Young's modulus and mass density of the beam at $\xi=0$, respectively; and the cross sectional area $A$ and moment of inertia $I$ can be expressed as:

$$
\begin{aligned}
A(\xi) & =A_{0}\left(1-c_{b} \xi\right)\left(1-c_{h} \xi\right), \\
I(\xi) & =A_{0}\left(1-c_{b} \xi\right)\left(1-c_{h} \xi\right)^{3}, \quad \xi \in[0,1] ;
\end{aligned}
$$

where $c_{b}$ is the breadth and $c_{h}$ is the height taper ratios, respectively, which must satisfy $0 \leq c_{b}, c_{h} \leq 1$ to insure that $A(\xi)$ and $I(\xi)$ are positive. Taking advantage of the shape functions of homogeneous uniform beam elements, Shahba et al. have investigated the free vibration of such axially functionally graded tapered Euler-Bernoulli beams through exploiting finite element method. ${ }^{23}$ The numerical results of non-dimensional natural frequency parameter $\Omega_{n}=\omega L^{2} \sqrt{\rho_{0} A_{0} / E_{0} I_{0}}$ of first mode are evaluated for $\mathrm{C}-\mathrm{C}$ beams and those together with other existing results are listed in Table 5. From Table 5, it is seen that our results clearly agree with the existing results by using the finite element method. ${ }^{23}$ We can also find that the results of the first mode natural frequencies decrease with an increase in the cross sectional parameters $c_{b}$ (or $c_{h}$ ). However, the effect of height tapering on non-dimensional natural frequencies is greater than that of breadth tapering. This is due to the fact that the effect of the former on moment of inertia is greater than the latter.

\section{CONCLUSIONS}

A new and simple approach has been presented to exactly determine the natural frequencies of Euler-Bernoulli beams with axially varying flexural rigidity and mass density. By expanding the mode shapes as a power series, we directly get corresponding characteristic equations in natural frequencies for all kinds of boundary conditions. An advantage of the suggested approach is that the derived characteristic equation is a polynomial equation, where the lower and higher-order natural frequencies can be determined simultaneously from the multi-roots by using commercial software. The effectiveness of the method has been confirmed by comparing our numerical results with the the exact ones and other numerical results available for homogeneous and nonhomogeneous beams. It has been seen that our numerical results show excellent consistency with the existing results. Furthermore, we have investigated the free vibration of axially graded and nonuniform beams to illustrate the effects of the gradient parameter and cross section on the natural frequencies under various end supports. This method is still efficient for treating similar stability problems of axially graded tapered Euler-Bernoulli beams.

\section{ACKNOWLEDGEMENTS}

The authors would like to give their thanks to the two reviewers for their useful suggestions to improve the quality of the manuscript. $\mathrm{YH}$ appreciates the financial support of the Funds of the Natural Science Foundation of Guangdong Province (Grant No. S2013010012463) and Excellent Teacher Scheme in Guangdong Higher Education Institutions (Grant No. Yq2014332). HWR appreciates the support of the Funds of the Guangdong college discipline construction (Grant No. 2013B020314020, No. 2014KZDXM063). 


\section{REFERENCES}

1 Abrate, S. Vibration of non-uniform rods and beams, Journal of Sound and Vibration, 185 (4), 703-716, (1995). http://dx.doi.org/10.1006/jsvi.1995.0410

2 Ece, N. C., Aydogdu, M., and Taskin, V. Vibration of a variable cross-section beam, Mechanics Research Communications, 34 (1), 78-84, (2007). http://dx.doi.org/10.1016/j.mechrescom.2006.06.005

3 Nachum, S. and Altus, E. Natural frequencies and mode shapes of deterministic and stochastic non-homogeneous rods and beams, Journal of Sound and Vibration, 302 (4-5), 903-924, (2007). http://dx.doi.org/10.1016/j.jsv.2006.12.021

${ }^{4}$ Hsu, J. C., Lai, H. Y., and Chen, C. K. Free vibration of nonuniform Euler-Bernoulli beams with general elastically end constraints using adomian modified decomposition method, Journal of Sound and Vibration, 318 (4-5), 965-981, (2008). http://dx.doi.org/10.1016/j.jsv.2008.05.010

5 Singh, K. V., Li, G., and Pang, S. S. Free vibration and physical parameter identification of non-uniform composite beams, Composite Structures, 74 (1), 37-50, (2006). http://dx.doi.org/10.1016/j.compstruct.2005.03.015

6 Alshorbagy, A. E., Eltaher, M. A., and Mahmoud, F. F. Free vibration characteristics of a functionally graded beam by finite element method, Applied Mathematical Modelling, 35 (1), 412-425, (2011). http://dx.doi.org/10.1016/j.apm.2010.07.006

7 Ozturk, B. and Coskun, S. B. The Homotopy Perturbation Method for free vibration analysis of beam on elastic foundation, Structural Engineering and Mechanics, 37 (4), 415425, (2011). http://dx.doi.org/10.12989/sem.2011.37.4.415

8 Atay, M. T. and Coskun, S. B. Elastic stability of Euler columns with a continuous elastic restraint using variational iteration method, Computers \& Mathematics with Applications, 58 (11-12), 2528-2534, (2009). http://dx.doi.org/10.1016/j.camwa.2009.03.051

9 Coskun, S. B. and Atay, M. T. Determination of critical buckling load for elastic columns of constant and variable cross-sections using variational iteration method, Computers \& Mathematics with Applications, 58 (11-12), 2260-2266, (2009). http://dx.doi.org/10.1016/j.camwa.2009.03.072

10 Atay, M. T. Determination of buckling loads of tilted buckled column with varying flexural rigidity using variational iteration method, International Journal of Nonlinear Sciences and Numerical Simulation, 11 (2), 93-103, (2010). http://dx.doi.org/10.1515/ijnsns.2010.11.2.97

11 Elishakoff, I. and Candan, S. Apparently first closedform solutions for vibrating inhomogeneous beams, International Journal of Solids and Structures, 38 (19), 3411-3441, (2001). http://dx.doi.org/10.1016/s00207683(00)00266-3
12 Elishakoff, I. and Candan, S. Apparently first closedform solution for frequencies of deterministically and/or stochastically inhomogeneous simply supported beams, Journal of Applied Mechanics, 68 (2), 176-185, (2001). http://dx.doi.org/10.1115/1.1355034

13 Elishakoff, I. and Guede, Z. Analytical polynomial solutions for vibrating axially graded beams, Mechanics of Advanced Materials and Structures, 11 (6), 517-533, (2004). http://dx.doi.org/10.1080/15376490490452669

14 Calio, I. and Elishakoff, I. Closed-form solutions for axially graded beam-columns, Journal of Sound and Vibration, 280 (3-5), 1083-1094, (2005). http://dx.doi.org/10.1016/j.jsv.2004.02.018

15 Aydogdu, M. Semi-inverse method for vibration and buckling of axially functionally graded beams, Journal of Reinforced Plastics and Composites, 27 (7), 683-691, (2008). http://dx.doi.org/10.1177/0731684407081369

16 Catal, S. Solution of free vibration equations of beam on elastic soil by using differential transform method, $A p$ plied Mathematical Modelling, 32(9), 1744-1757, (2008). http://dx.doi.org/10.1016/j.apm.2007.06.010

17 Shahba, A. and Rajasekaran, S. Free vibration and stability of tapered Euler-Bernoulli beams made of axially functionally graded materials, Applied Mathematical Modelling, 36 (7), 3094-3111, (2012). http://dx.doi.org/10.1016/j.apm.2011.09.073

18 Hein, H. and Feklistova, L. Free vibrations of nonuniform and axially functionally graded beams using Haar wavelets, Engineering Structures, 33 (12), 3696-3701, (2011). http://dx.doi.org/10.1016/j.engstruct.2011.08.006

19 Shahba, A., Attarnejad, R., Tavanaie Marvi, M., and Hajilar, S. Free vibration and stability analysis of axially functionally graded tapered Timoshenko Beams with classical and non-classical boundary conditions, Composites B, 42 (4), 801-808, (2011). http://dx.doi.org/10.1016/j.compositesb.2011.01.017

${ }^{20} \mathrm{Li}, \mathrm{X}$. F., Kang, Y. A., and Wu, J. X. Exact frequency equations of free vibration of exponentially functionally graded beams, Applied Acoustics, 74 (3), 413-420, (2013). http://dx.doi.org/10.1016/j.apacoust.2012.08.003

21 Huang, Y. and Luo, Q. Z. A simple method to determine the critical buckling loads for axially inhomogeneous beams with elastic restraint, Computers \& Mathematics with Applications, 61 (9), 2510-2517, (2011). http://dx.doi.org/10.1016/j.camwa.2011.02.037

22 Weaver, W., Timoshenko, S., and Young, D. H. Vibration problems in engineering, Wiley-Interscience, New York, (1990).

23 Shahba, A., Attarnejad, R., and Hajilar, S. Free vibration and stability of axially functionally graded tapered EulerBernoulli beams, Shock and Vibration, 18 (5), 683-696, (2011). http://dx.doi.org/10.1155/2011/591716 